\title{
Unilateral versus bilateral anterograde cerebral perfusion in acute type A aortic dissection repair: A systematic review and meta-analysis
}

\author{
Panagiotis T. Tasoudis ${ }^{1}$, Dimitrios N. Varvoglis ${ }^{1}$, Evangelos Vitkos ${ }^{2}$, John Ikonomidis ${ }^{3}$, \\ and Thanos Athanasiou ${ }^{1}$ \\ ${ }^{1}$ Panepistemio Thessalias Tmema Iatrikes \\ ${ }^{2}$ Surgery Working Group Society of Junior Doctors Athens Greece \\ ${ }^{3}$ University of North Carolina at Chapel Hill Department of Surgery
}

December 18, 2021

\begin{abstract}
Objectives: The aim of the study is to compare the safety and efficacy of unilateral anterograde cerebral perfusion (UACP) and bilateral anterograde cerebral perfusion (BACP) for acute type A aortic dissection (ATAAD). Methods: A systematic review of MEDLINE (PubMed), Scopus, and Cochrane Library databases (last search: August $7^{\text {th }}, 2021$ ) was performed according to the PRISMA statement. Studies directly comparing UACP versus BACP for ATAAD were included. Random-effects meta-analyses were performed. Results: Eight retrospective cohort studies were identified, incorporating 2416 patients (UACP: 843, BACP: 1573). No statistically significant difference was observed regarding in-hospital mortality (odds ratio [OR]:1.05 [95\% Confidence Interval (95\%CI):0.70-1.57]), permanent neurological deficit (PND) (OR: 0.94 [95\%CI:0.52-1.70]), transient neurological deficit (TND) (OR: 1.37 [95\%CI:0.98-1.92]), renal failure (OR: 0.96 [95\%CI:0.70-1.32]), and re-exploration for bleeding (OR: 0.77 [95\%CI:0.48-1.22]). Meta-regression analysis revealed that PND and TND were not influenced by differences in rates of total arch repair, Bentall procedure and concomitant CABG in UACP and BACP groups. Cardiopulmonary bypass time (Standard Mean Difference [SMD]:-0.11 [95\%CI:-0.22, 0.44]), Cross clamp time (SMD:-0.04 [95\%CI:-0.38, 0.29]) and hypothermic circulatory arrest time (SMD:-0.12 [95\%CI:-0.55, 0.30]) were comparable between UACP and BACP. Intensive care unit stay was shorter in BACP arm (SMD:0.16 [95\%CI:0.01, 0.31]), however, length of hospital stay was shorter in UACP arm (SMD:-0.25 [95\%CI:-0.45, -0.06]). Conclusions: UACP and BACP had similar results in terms of in-hospital mortality, PND, TND, renal failure and re-exploration for bleeding rate in patients with ATAAD. ICU stay was shorter in the BACP arm while LOS was shorter in the UACP arm.
\end{abstract}

Title: Unilateral versus bilateral anterograde cerebral perfusion in acute type $\mathbf{A}$ aortic dissection repair: A systematic review and meta-analysis

Authors: Panagiotis T. Tasoudis ${ }^{1}$, Dimitrios N. Varvoglis ${ }^{2}$, Evangelos Vitkos ${ }^{3}$, John S. Ikonomidis ${ }^{4}$, Thanos Athanasiou ${ }^{5}$

Author Affiliations:

1. Panagiotis T. Tasoudis, MS, Department of Cardiothoracic Surgery, Faculty of Medicine, School of Health Sciences, University of Thessaly, Biopolis, Larissa, Greece; Surgery Working Group, Society of Junior Doctors, Athens, Greece. Email:tasoudis@gmail.com

2. Dimitrios N. Varvoglis, MS, Department of Cardiothoracic Surgery, Faculty of Medicine, School of Health Sciences, University of Thessaly, Biopolis, Larissa, Greece; Surgery Working Group, Society of Junior Doctors, Athens, Greece. Email:dvarvoglis97@gmail.com 
3. Evangelos Vitkos, DDS, MD, Surgery Working Group, Society of Junior Doctors, Athens, Greece. Email:envitkos@gmail.com

4. John S. Ikonomidis MD, PhD, Professor of Surgery, Chief, Division of Cardiothoracic Surgery, Department of Surgery, School of Medicine, University of North Carolina at Chapel Hill Chapel Hill, NC. Email:john_ikonomidis@med.unc.edu

5. Thanos Athanasiou, MD, PhD, MBA, FECTS, FRCS, Department of Surgery and Cancer, Imperial College London, St Mary's Hospital, London, W2 1NY, UK, Department of Cardiothoracic Surgery, University Hospital of Larissa, Biopolis, Larissa, Greece, 41110. Email:t.athanasiou@imperial.ac.uk

Correspondence to: Panagiotis T. Tasoudis,tasoudis@gmail.comDepartment of Cardiothoracic Surgery, University Hospital of Larissa, Biopolis, Larissa, Greece, 41110, Tel:+306986186798

\section{Conflicts of Interest}

The authors declare no conflicts of interest.

\section{Funding}

None

\section{Ethical Approval}

Does not apply.

\section{Informed Consent}

Does not apply.

\section{Acknowledgements}

None.

\section{Data availability statement}

The authors confirm that the data supporting the findings of this study are available within the article and its supplementary materials.

Authors' contributions: Study design: PTT, TA; Study conduct: PTT, DNV, EV; Data analysis: PTT; Data interpretation: PTT, DNV, EV; Manuscript drafting: PTT, DNV, JSI, TA; Writing and revising paper: PTT, JSI, TA

Running title: UACP vs. BACP in ATAAD: A meta-analysis

\section{Total Word count: 2482}

Key words: unilateral anterograde cerebral perfusion, bilateral anterograde cerebral perfusion, acute type A aortic dissection

\section{Glossary of Abbreviations:}

ACP: anterograde cerebral perfusion

UACP: unilateral anterograde cerebral perfusion

BACP: bilateral anterograde cerebral perfusion

ATAAD: acute type A aortic dissection

HCA: hypothermic circulatory arrest

PND: permanent neurological deficit

TND: transient neurological deficit 
LOS: length of hospital stay

ICU: intensive care unit

CPB: cardiopulmonary bypass

CCP: cross clamp time

TAR: total arch repair

OR: odds ratio

CI: confidence interval

SMD: standard mean difference

PRISMA: Preferred Reporting Items for Systematic Reviews and Meta-Analyses

PICO : Population/Participants, Intervention, Comparison, and Outcome

Central Picture Legend: Unilateral Vs Bilateral Anterograde Cerebral Perfusion for Acute type A aortic dissection

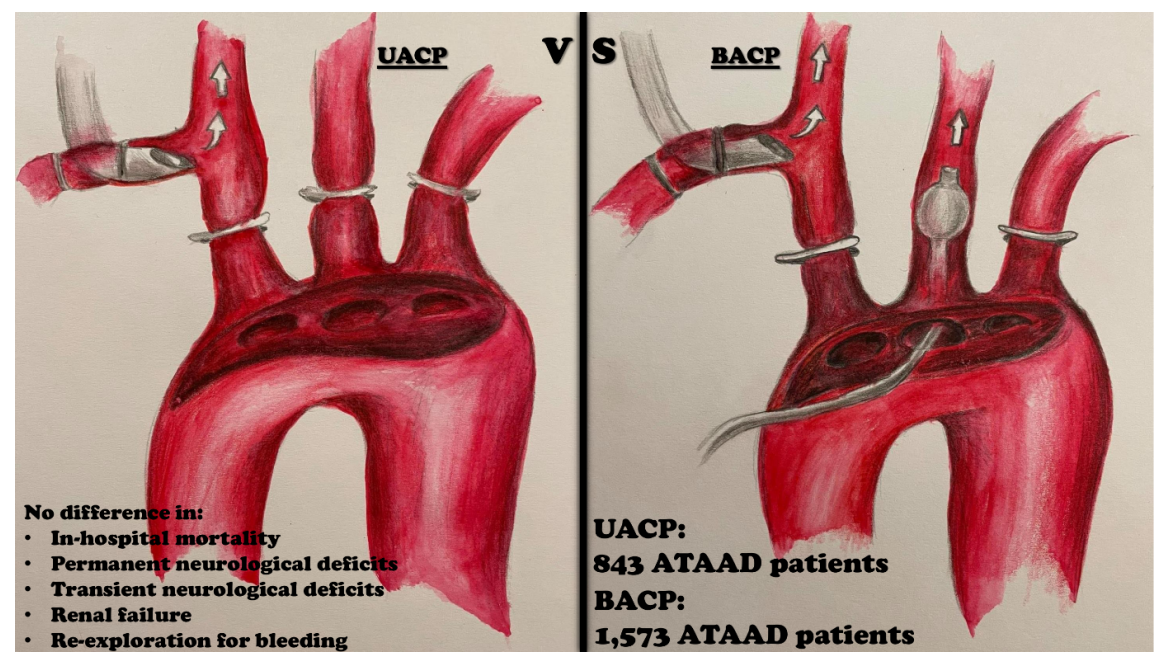

Central Message: UACP and BACP had similar results regarding in-hospital mortality, PND, TND, renal failure, and re-exploration for bleeding. ICU stay was shorter in the BACP arm while LOS was shorter in the UACP arm

Perspective statement: UACP and BACP are comparable regarding the post-operative outcomes in patients with ATAAD, thus utilization should be tailored to the surgeon's experience and requires individualized patient selection considering the duration of the operation and the anatomy of the supra-aortic vessels. Implementation of the available pre-operative and intra-operative means could facilitate the decision process.

Abstract

Objectives: The aim of the study is to compare the safety and efficacy of unilateral anterograde cerebral perfusion (UACP) and bilateral anterograde cerebral perfusion (BACP) for acute type A aortic dissection (ATAAD).

Methods: A systematic review of the MEDLINE (PubMed), Scopus, and Cochrane Library databases (last search: August $7^{\text {th }}, 2021$ ) was performed according to the PRISMA statement. Studies directly comparing UACP versus BACP for ATAAD were included. Random-effects meta-analyses were performed. 
Results: Eight retrospective cohort studies were identified, incorporating 2416 patients (UACP: 843, BACP: 1573). No statistically significant difference was observed regarding in-hospital mortality (odds ratio $[\mathrm{OR}]: 1.05$ [95\% Confidence Interval (95\%CI):0.70-1.57]), permanent neurological deficit (PND) (OR: 0.94 [95\%CI:0.52-1.70]), transient neurological deficit (TND) (OR: 1.37 [95\%CI:0.98-1.92]), renal failure (OR: 0.96 [95\%CI:0.70-1.32]), and re-exploration for bleeding (OR: 0.77 [95\%CI:0.48-1.22]). Meta-regression analysis revealed that PND and TND were not influenced by differences in rates of total arch repair, Bentall procedure and concomitant CABG in UACP and BACP groups. Cardiopulmonary bypass time (Standard Mean Difference [SMD]:-0.11 [95\%CI:-0.22, 0.44]), Cross clamp time (SMD:-0.04 [95\%CI:-0.38, 0.29]) and hypothermic circulatory arrest time (SMD:-0.12 [95\%CI:-0.55, 0.30]) were comparable between UACP and BACP. Intensive care unit stay was shorter in BACP arm (SMD:0.16 [95\%CI:0.01, 0.31]), however, length of hospital stay was shorter in UACP arm (SMD:-0.25 [95\%CI:-0.45, -0.06]).

Conclusions: UACP and BACP had similar results in terms of in-hospital mortality, PND, TND, renal failure and re-exploration for bleeding rate in patients with ATAAD. ICU stay was shorter in the BACP arm while LOS was shorter in the UACP arm.

\section{Word Count: 250}

\section{Introduction}

Acute onset dissections involving the ascending arch are classified as acute type A aortic dissection $(\text { ATAAD })^{1}$. Treatment modalities for this condition have been improved over the years, yet mortality and morbidity rates remain elevated ${ }^{2,3}$. In recent years the implementation of hypothermic circulatory arrest (HCA) along with anterograde cerebral perfusion (ACP) has decreased the numbers of post-operative neurological deficits ${ }^{4}$. Nevertheless, there is an ongoing debate on which ACP technique should be implemented ${ }^{5}$. Unilateral anterograde cerebral perfusion (UACP) implies less manipulation of the vessels ${ }^{6,7}$, however the protection offered to the contralateral cerebral hemisphere is debatable ${ }^{8}$. Bilateral anterograde cerebral perfusion BACP is considered a safer option when the duration of ACP is long and is the technique most utilized by aortic surgeons ${ }^{9}$. Yet, the risk of dislodging a clot and causing an embolic stroke remains one of the biggest disadvantages of this cerebral protection modality ${ }^{10}$.

Previous meta-analyses on the topic included non-comparative studies and patients with heterogenous aortic pathologies which implies poor quality of evidence ${ }^{11-13}$. Moreover, all previous meta-analyses are outdated and inconclusive regarding the optimal cerebral protection method ${ }^{11-13}$. Accounting our inability to draw conclusions using current data along with the emergence of new relevant studies, we conducted a systematic review and meta-analysis to assess the preoperative and intraoperative characteristics as well as the early postoperative outcomes of patients, on whom UACP versus BACP was implemented when being surgically treated for ATAAD.

\section{Material and methods}

\section{Study design and inclusion/exclusion criteria}

The research team complied with the PRISMA (Preferred Reporting Items for Systematic Reviews and Meta-Analysis) guidelines for this systematic review and meta-analysis. The research question was defined via the PICO (Population/Participants, Intervention, Comparison, and Outcome) criteria:

1. Population/ Participants: Patients undergoing surgery for ATAAD

2. Intervention: UACP

3. Comparison: BACP

4. Outcomes: Primary outcomes were in-hospital mortality, defined as mortality within thirty days after surgery; post-operative permanent neurological deficits (PND), defined as neurological deficits that persisted after discharge, and post-operative transient neurological deficits (TND), defined as neurological deficits that resolved during hospitalization. Secondary outcomes were the length of hospital stay (LOS); the length of intensive care unit (ICU) stay; renal failure, defined as the post-operative 
need for hemodialysis; re-exploration for bleeding, defined as any invasive procedure performed due to hemodynamic instability.

All comparative studies written in English that reported on patients with ATAAD undergoing surgery that implemented either UACP or BACP, were deemed eligible. Studies were excluded using the following criteria: all the studies not published in English, non-comparative studies, meta-analyses and systematic reviews, editorials, letters to the editor, comments, personal opinions, errata, case series and case reports, studies that reported on patients with heterogenous aortic pathologies. From studies with overlapping populations only one study was selected.

\section{Search strategy}

MEDLINE (via PubMed), Scopus and Cochrane library were searched (last search August $7^{\text {th }}, 2021$ ) using the following algorithm: ((Cerebral Perfusion) OR (Cerebral protection) OR (Brain protection) OR (ACP) OR (SACP)) AND ((ATAAD) OR (Acute Type A Aortic Dissection) OR (DeBakey type I) OR (DeBakey type II)). Title and abstract screening and full text eligibility were performed by two independent investigators (DNV, ENV). A third reviewer (PTT) was consulted when there was a conflict. A thorough search of potentially eligible articles was made via the snowball method ${ }^{14}$.

\section{Data extraction and tabulation}

Data was extracted by two independent investigators (DNV, ENV) and disagreements reached consensus via discussion with a third investigator (PTT). Data were inserted into a predesigned table for evidence synthesis. The following data were extracted: study characteristics (first author, year of publication, study design, study center, study period, number of patients); patients baseline characteristics (age in years, gender and various comorbidities); perioperative characteristics (cardiopulmonary bypass [CPB] time, cross clamp $[\mathrm{CCP}]$ time, UACP time, UACP time, hypothermic circulatory arrest [HCA] duration and concomitant procedures); post-operative outcomes (mortality, PND and TND rates, renal failure rates, LOS, length of ICU stay and re-exploration for bleeding)

\section{Quality of evidence assessment}

Eligible studies' quality was assessed by the implementation of the $\mathrm{NOS}^{15}$. A study that received a grading of at least 6 or more was considered of high quality. Since all the assessed outcomes are post-operative outcomes, all included studies were deemed adequate regarding the follow up period.

\section{Statistical analysis}

We conducted meta-analyses to compare the outcomes of UACP versus BACP as a cerebral protective strategy in patients undergoing surgery for ATAAD. Continuous variables were analyzed using standardized mean difference (SMD) and 95\% confidence intervals (95\% CI). A SMD greater than zero corresponded to larger values in the UACP arm. Categorical values were analyzed using odds ratio (OR) and 95\% CI. An OR greater than 1 indicated that the outcome was more frequently present in the UACP arm. Inherent clinical heterogeneity between the studies was balanced via the implementation of random effects models (DerSimonian-Laird). Results were displayed in forest plots.

Pre-specified random effects meta-regression analyses were conducted to examine the impact of moderator variables on outcomes. Specifically, using this technique we attempted to assess the effect of total arch repair (TAR), implementation of Bentall procedure and performing concomitant coronary artery bypass graft (CABG) during the surgical repair of ATAAD on PND and TND.

Between-study statistical heterogeneity was assessed with the Cochran Q statistic and by estimating $I^{2}$ . High heterogeneity was confirmed with a significance level of $\mathrm{p}<0.10$ and $I^{2}$ of at least $50 \%$ or more. Publication bias was assessed via funnel plots and Eggers' test for each outcome of interest and $\mathrm{p}<0.10$ was considered statistically significant. All analyses were performed using STATA IC16.0 (StataCorp LLC, College Station, Texas). 
1.

2 .

3. Results

4. Study selection and baseline characteristics

Through the systematic search we conducted, a total of 1183 articles were retrieved. Detailed study selection process is presented inSupplemental Figure 1 . Ultimately, 8 studies reporting on cumulatively 2416 patients, 843 patients undergoing UACP and 1573 patients undergoing BACP were included in this metaanalysis ${ }^{6,7,16-21}$. Study characteristics are reported in Table 1 . Patient baseline and clinical characteristics are cumulatively reported intraoperative characteristics in Table 2. All outcomes are cumulatively presented inSupplemental table 1 .

\section{Intraoperative characteristics}

CCP time was reported in 7 studies $^{6,16-21}$ and no difference was noted between UACP and BACP groups (SMD: -0.04, 95\%CI: $[-0.38]-[0.29], p=0.79, \mathrm{I}^{2}=91.29 \%$ ). $\mathrm{CPB}$ duration was reported in 7 studies $^{6,7,16,17,19-21}$. CPB time did not differ significantly between the groups (SMD: $0.11,95 \% \mathrm{CI}$ : [-0.22]

- $\left.[0.44], p=0.52, \mathrm{I}^{2}=89.65 \%\right)$. Regarding HCA duration, meta-analysis of all included studies demonstrated that there was no difference between the two groups (SMD: $-0.12,95 \%$ CI: $[-0.55]-[0.30], p=0.57$, $\left.\mathrm{I}^{2}=95.10 \%\right)$. All included studies implemented deep $\left(<24^{\circ} \mathrm{C}\right)$ or moderate hypothermia $\left(>24^{\circ} \mathrm{C}\right)$ during aortic repair, however further details were not available.

\section{Primary outcomes}

\section{In-hospital mortality}

In- hospital mortality was reported in all included studies. Overall, there were $96(11.4 \%, \mathrm{n}=96 / 1275)$ inhospital mortalities in the UACP group and $289(18,4 \%, \mathrm{n}=289 / 1486)$ in the BACP group during the hospital stay. No statistically significant difference was observed between the UACP group and BACP group in terms of in-hospital mortality (OR:1.05, 95\%CI [0.70] - [1.57], $p=0.80, \mathrm{I}^{2}=39.58 \%$ ). (Figure 1)

PND

PND events were reported in all studies. A total of 87 patients were reported with PND in the UACP group $(10.3 \%, \mathrm{n}=87 / 843)$ and 196 patients in the BACP group $(12.5 \%, \mathrm{n}=196 / 1573)$. The two groups were comparable in terms of PND (OR: 0.94, 95\%CI: [0.52] - [1.70], $p=0.84, \mathrm{I}^{2}=69.34 \%$ ). (Figure 2.A)

\section{TND}

TND events were reported in seven included studies $6,7,16,17,19-21$. Overall, 85 patients presented TND in the UACP group $(11.7 \%, \mathrm{n}=85 / 726)$ and 76 patients in the BACP group $(9.3 \%, \mathrm{n}=76 / 813)$. The two groups were comparable regarding TND (OR: $1.37,95 \%$ CI: $[0.98]-[1.92], p=0.07, \mathrm{I}^{2}=0.0 \%$ ). (Figure 2.B)

\section{Secondary outcomes}

\section{LOS}

LOS was reported in four studies $6,7,19,20$. UACP was associated with decreased LOS compared to BACP (SMD: $-0.25,95 \%$ CI: $[-0.45]-[-0.06], p=0.01, \mathrm{I}^{2}=54.39 \%$ ).

\section{ICU stay}

ICU stay duration was reported in five studies $7,16,17,19,20$. UACP demonstrated increased ICU stay length compared to BACP (SMD: 0.16, 95\%CI: [0.01] - [0.31], $p=0.04, \mathrm{I}^{2}=28.56 \%$ ).

\section{Renal failure}


Renal failure events were available in all included studies. There was no statistically significant difference between the UACP group and the BACP group in terms of renal failure (OR: 0.96, 95\%CI: [0.70] - [1.32], $p=0.80, \mathrm{I}^{2}=20.39 \%$ ).

\section{Re-exploration for bleeding}

Re-exploration for bleeding was available in 6 studies $^{6,7,17-20}$. No statistically significant difference was observed between the UACP group and the BACP group in terms of re-exploration for bleeding (OR: 0.77, 95\%CI: $\left.[0.48]-[1.22] . p=0.27, \mathrm{I}^{2}=18.53 \%\right)$.

\section{Meta-regression analysis}

Random-effects meta-regression analysis was performed to examine potential relationships between PND or TND and TAR. Data separating patients with ATAAD undergoing TAR or hemiarch repair were reported in seven sudies. Implementation of TAR was expressed as difference of rate of occurrence in the UACP versus BACP groups. Meta-regression analysis revealed that TAR had no statistically significant influence on PND $(p=0.49)$ (Supplemental Figure 2) nor on TND $(p=0.91)$.

Additional meta-regression analyses were conducted to explore a potential influence of Bentall procedure or CABG on both PND and TND. Utilization of Bentall procedure or CABG was expressed as a difference of rate of occurrence in the UACP versus BACP groups. Meta-regression analysis revealed that Bentall procedure and CABG had no statistically significant influence on PND and TND (Supplemental Table 2) .

\section{Quality of evidence and publication bias assessment}

All included studies were retrospective cohorts and thus for quality assessment we utilized the NOS. Detailed NOS quality assessment for each of the eligible studies is shown in Supplemental table 3.In addition, statistically significant heterogeneity was found in PND rate, CPB time, CCP time and HCA time (Supplemental Table 1) .

Egger's test revealed no publication bias in the funnel plots of most studied outcomes, except for CPB time $(p<0.01)$, CCP time $(p<0.01)$, and HCA time $(p=0.02)$ (Supplemental Figure 3) .

\section{Conclusions}

The outcomes of the present systematic review and meta-analysis indicate that UACP and BACP are comparable in terms of in-hospital mortality, PND, TND, renal failure, and re-exploration for bleeding. Particularly for PND and TND, our meta-regression analysis showed that the proportion of patients who underwent TAR, Bentall procedure and concomitant CABG during ATAAD repair in each arm of our study, did not significantly influence the rate of PND and TND events. The two cerebral protection techniques were also similar regarding $\mathrm{CPB}$ time, CCP time and HCA time. However, ICU stay was found to be borderline increased in the UACP group, while the LOS was found to be increased in the BACP group.

In contrast to previous meta-analyses which included both comparative and single arm studies as well as studies reporting on patients with heterogenous aortic pathologies ${ }^{11-13}$, we incorporated only comparative studies reporting solely on patients with ATTAD to limit potential confounding biases. It should also be noted that, except for one study ${ }^{7}$, all our individual studies were published after 2017 and consequently were not included in the previous meta-analyses. Yet, our findings are in accordance with the findings of these previous reports ${ }^{11-13}$. Moreover, our cumulative results were consistent with the corresponding results in the included individual studies.

Aiming to reveal potential factors influencing our results, we examined through meta-regression analysis the effect of TAR, Bentall procedure and concomitant CABG on the PND and TND incidence. Additionally, longer circulatory arrest time was associated with increased risk for mortality and PND in patients operated with UACP compared to $\mathrm{BACP}^{12,13}$. Therefore, to further limit potential confounders, we compared the intra-operative characteristics of the incorporated studies. Cumulatively, no differences were noted in terms 
$\mathrm{CPB}, \mathrm{CCP}$ and $\mathrm{HCA}$ times and thus further analysis on the impact of these factors on our findings was deferred.

For post-operative patient care, our analysis revealed that UACP offers marginally increased length of ICU. On the other hand, LOS was found to be longer in BACP arm. Accounting that BACP is a more demanding procedure where more vessels are manipulated and anastomosed ${ }^{19,21}$, it is logical to assume that patients would require longer periods for recovery and thus longer LOS. These findings, however, should be interpreted cautiously since ICU and LOS were reported only in few studies and displayed considerable heterogeneity.

Nevertheless, while UACP is supported by available evidence to provide equivalent results compared to $\mathrm{BACP}$, most aortic surgeons insist on opting for BACP ${ }^{9}$. UACP's efficacy was considered to be inextricably connected to the patency of the circle of Willis, in order to maintain adequate blood supply to the contralateral hemisphere. It is true that anatomic variations on the circle of Willis are very common in the population, however, studies assessing UACP in patients with incomplete circle of Willis reported that the incidence of neurological adverse events was not affected by the anatomy of the circle of Willis ${ }^{22,23}$. This finding could be explained by the fact that in UACP contralateral cerebral perfusion can be facilitated by extracranial vessels, and that watershed cerebral infarcts have various clinical presentations and are not easily diagnosed and documented. On the other hand, BACP increases the risk for clot dislodging and air embolism in the cerebral circulation, due to excess manipulation and cannulation of the supra-aortic vessels ${ }^{6,19}$. Additionally, in patients with aortic dissections, manipulation of the fragile pathologic vessel wall imposes the risk of vessel rapture and could lead to catastrophic complications ${ }^{11}$.

Transcranial doppler of the middle cerebral artery, CT angiography of the circle of Willis, near-infrared spectroscopy (NIRS) and blood backflow from left common carotid or subclavian artery are some of the pre-operative and intra-operative techniques used to facilitate the selection of the optimal cerebral perfusion modality $^{7,24}$. NIRS is used to provide information regarding brain's perfusion intra-operatively, and some surgeons recommend switching UACP to BACP intraoperatively when NIRS suggests decreased cerebral perfusion ${ }^{7}$. However, in cases of ATAAD there is limited time for pre-operative assessment. Additionally, NIRS provides information about the perfusion of few brain areas while the brainstem along with other parts of the brain are not assessed ${ }^{11}$. These barriers render the selection of the proper cerebral perfusion modality in patients with ATAAD very challenging.

Accounting that both UACP and BACP are comparable regarding the post-operative outcomes in patients with ATAAD, we conclude that the utilization of BACP over UACP should be tailored according to the surgeon's and the center's experience and requires individualized patient selection considering the anticipated duration of the operation as well as the anatomy and the integrity of the patient's supra-aortic vessels. Implementation of the available pre-operative and intra-operative means could also decisively influence the appropriate cerebral perfusion technique for each individual case.

\section{Limitations}

There are certain limitations in this study that should be acknowledged. First, all studies were nonrandomized and retrospective in nature and thus impart the risk of selection bias. Additionally, since this study is a meta-analysis, we didn't have access to individual patient data. Moreover, not all reports contained all variables of interest and there were differences in the definitions of the reported items between the included studies, thus relative rates were estimated based on the availability of data. Finally, the procedures in the included studies were performed in various centers by different surgeons and this could have confounded our results.

\section{References}

1. LeMaire SA, Russell L. Epidemiology of thoracic aortic dissection.Nat Rev Cardiol . 2011;8:103-113. doi: $10.1038 /$ nrcardio.2010.187

2. Larsen M, Trimarchi S, Patel HJ, et al. Extended versus limited arch replacement in acute Type A aortic dissection. Eur J Cardiothorac Surg . 2017;52:1104-1110. doi:10.1093/ejcts/ezx214 
3. Abe T, Yamamoto H, Miyata H, et al. Patient trends and outcomes of surgery for type A acute aortic dissection in Japan: an analysis of more than 10000 patients from the Japan Cardiovascular Surgery Database.Eur J Cardiothorac Surg . 2020;57:660-667. doi:10.1093/ejcts/ezz323

4. Zierer A, El-Sayed Ahmad A, Papadopoulos N, Moritz A, Diegeler A, Urbanski PP. Selective antegrade cerebral perfusion and mild $\left(28^{\circ} \mathrm{C}-30^{\circ} \mathrm{C}\right)$ systemic hypothermic circulatory arrest for aortic arch replacement: Results from 1002 patients. In: Journal of Thoracic and Cardiovascular Surgery. Vol 144. Mosby Inc.; 2012:1042-1050. doi:10.1016/j.jtcvs.2012.07.063

5. El-Sayed Ahmad A, Papadopoulos N, Risteski P, Moritz A, Zierer A. The Standardized Concept of Moderate-to-Mild ([?]28degC) Systemic Hypothermia During Selective Antegrade Cerebral Perfusion for AllComers in Aortic Arch Surgery: Single-Center Experience in 587 Consecutive Patients Over a 15-Year Period. Ann Thorac Surg . 2017;104:49-55. doi:10.1016/j.athoracsur.2016.10.024

6. Norton EL, Wu X, Kim KM, Patel HJ, Deeb GM, Yang B. Unilateral is comparable to bilateral antegrade cerebral perfusion in acute type A aortic dissection repair. J Thorac Cardiovasc Surg . 2020;160:617-625.e5. doi:10.1016/j.jtcvs.2019.07.108

7. Preventza O, Simpson KH, Cooley DA, et al. Unilateral versus bilateral cerebral perfusion for acute type A aortic dissection.Ann Thorac Surg . 2015;99:80-87. doi:10.1016/j.athoracsur.2014.07.049

8. Malvindi PG, Scrascia G, Vitale N. Is unilateral antegrade cerebral perfusion equivalent to bilateral cerebral perfusion for patients undergoing aortic arch surgery? Interact Cardiovasc Thorac Surg . 2008;7:891897. doi:10.1510/icvts.2008.184184

9. De Paulis R, Czerny M, Weltert L, et al. Current trends in cannulation and neuroprotection during surgery of the aortic arch in Europe. Eur J Cardio-thoracic Surg . 2015;47:917-923. doi:10.1093/ejcts/ezu284

10. Leshnower BG, Myung RJ, Kilgo PD, et al. Moderate hypothermia and unilateral selective antegrade cerebral perfusion: a contemporary cerebral protection strategy for aortic arch surgery. Ann Thorac Surg . 2010;90:547-554. doi:10.1016/j.athoracsur.2010.03.118

11. Tian DH, Wilson-Smith A, Koo SK, Forrest P, Kiat H, Yan TD. Unilateral Versus Bilateral Antegrade Cerebral Perfusion: A Meta-Analysis of Comparative Studies. Hear Lung Circ . 2019;28:844-849. doi:10.1016/j.hlc.2019.01.010

12. Angeloni E, Melina G, Refice SK, et al. Unilateral versus bilateral antegrade cerebral protection during aortic surgery: An updated meta-analysis. Ann Thorac Surg . 2015;99:2024-2031. doi:10.1016/j.athoracsur.2015.01.070

13. Angeloni E, Benedetto U, Takkenberg JJM, et al. Unilateral versus bilateral antegrade cerebral protection during circulatory arrest in aortic surgery: A meta-analysis of 5100 patients. J Thorac Cardiovasc Surg . 2014;147:60-67. doi:10.1016/j.jtcvs.2012.10.029

14. Wohlin C. Guidelines for snowballing in systematic literature studies and a replication in software engineering. In: ACM International Conference Proceeding Series . EASE '14. ACM; 2014;38:138-10. doi:10.1145/2601248.2601268

15. Stang A. Critical evaluation of the Newcastle-Ottawa scale for the assessment of the quality of nonrandomized studies in meta-analyses.Eur J Epidemiol . 2010;25:603-605. doi:10.1007/s10654-010-9491-z

16. Liu Z, Wang C, Zhang X, Wu S, Fang C, Pang X. Effect of different types of cerebral perfusion for acute type A aortic dissection undergoing aortic arch procedure, unilateral versus bilateral. BMC Surg . 2020;20:1-8. doi:10.1186/s12893-020-00957-8

17. Dong SB, Xiong JX, Zhang K, et al. Different hypothermic and cerebral perfusion strategies in extended arch replacement for acute type a aortic dissection: A retrospective comparative study. J Cardiothorac Surg . 2020;15:1-8. doi:10.1186/s13019-020-01284-y 
18. Benedetto U, Dimagli A, Cooper G, et al. Neuroprotective strategies in acute aortic dissection: an analysis of the UK National Adult Cardiac Surgical Audit. Eur J Cardio-Thoracic Surg . 2021;00:1-8. doi:10.1093/ejcts/ezab192

19. Piperata A, Watanabe M, Pernot M, et al. Unilateral versus bilateral cerebral perfusion during aortic surgery for acute type A aortic dissection: a multicentre study. Eur J Cardio-Thoracic Surg . 2021;00:1-8. doi:10.1093/ejcts/ezab341

20. Tong G, Zhang B, Zhou X, et al. Bilateral versus unilateral antegrade cerebral perfusion in total arch replacement for type A aortic dissection. J Thorac Cardiovasc Surg . 2017;154:767-775. doi:10.1016/j.jtcvs.2017.02.053

21. Angleitner P, Stelzmueller ME, Mahr S, Kaider A, Laufer G, Ehrlich M. Bilateral or unilateral antegrade cerebral perfusion during surgery for acute type A dissection. J Thorac Cardiovasc Surg . 2020;159:21592167.e2. doi:10.1016/j.jtcvs.2019.06.057

22. Urbanski PP, Lenos A, Blume JC, et al. Does anatomical completeness of the circle of Willis correlate with sufficient cross-perfusion during unilateral cerebral perfusion? Eur J Cardio-thoracic Surg . 2008;33:402408. doi:10.1016/j.ejcts.2007.12.021

23. Papantchev V, Stoinova V, Aleksandrov A, et al. The role of willis circle variations during unilateral selective cerebral perfusion: A study of 500 circles. Eur J Cardio-thoracic Surg . 2013;44:743-753. doi:10.1093/ejcts/ezt103

24. Tian DH, Wilson-Smith A, Koo SK, Forrest P, Kiat H, Yan TD. Unilateral Versus Bilateral Antegrade Cerebral Perfusion: A Meta-Analysis of Comparative Studies. Heart Lung Circ . 2019;28:844-849. doi:10.1016/j.hlc.2019.01.010

\begin{tabular}{lllll}
\hline Author & Year & Center & Country & Design \\
\hline Preventza et al. & 2015 & Texas Heart Institute, Houston & USA & Retrospective Cohort \\
Guang et al. & 2017 & Guanzhou General Military Hospital, Guanzhou & China & Retrospective Cohort \\
Dong et al. & 2020 & Beijing Anhen Hospital, Beijing & China & Retrospective Cohort \\
Liu et al. & 2020 & Qilu Hospital of Shandong University, Jinan & China & Retrospective Cohort \\
Norton et al. & 2020 & University of Michigan, Michigan & USA & Retrospective Cohort \\
Angleitner et al. & 2020 & Medical University of Vienna, Vienna & Austria & Retrospective Cohort \\
Piperata et al. & 2021 & Multicenter Study & Italy, France, Japan & Retrospective Cohort \\
Benedetto et al. & 2021 & Multicenter Study & United Kingdom & Retrospective Cohort \\
\hline
\end{tabular}

Table 1. Study characteristics

$\mathrm{UACP}=$ unilateral anterograde cerebral perfusion; BACP $=$ bilateral anterograde cerebral perfusion

Table 2. Cumulative patient baseline characteristics of the studies included in the meta-analysis.

\begin{tabular}{lll}
\hline & UACP $(\mathbf{n}=\mathbf{8 4 3})$ & BACP $(\mathbf{n}=\mathbf{1 5 7 3})$ \\
\hline $\begin{array}{l}\text { Demographic } \\
\text { characteristics }\end{array}$ & \\
$\begin{array}{l}\text { Age (years) } \\
\text { Female }\end{array}$ & $59.5 \pm 14.2(\mathrm{n}=843)$ & $60.2 \pm 13.8(\mathrm{n}=1573)$ \\
Male & $38.6 \%(\mathrm{n}=325 / 843)$ & $35.3 \%(\mathrm{n}=555 / 1573)$ \\
Comorbidities & $61.4 \%(\mathrm{n}=518 / 843)$ & $64.7 \%(\mathrm{n}=1018 / 1573)$ \\
Hypertension & & $77.7 \%(\mathrm{n}=632 / 813)$ \\
$\begin{array}{l}\text { Diabetes } \\
\text { Marfan Syndrome }\end{array}$ & $80.0 \%(\mathrm{n}=581 / 726)$ & $9.3 \%(\mathrm{n}=146 / 1573)$ \\
& $9.7 \%(\mathrm{n}=82 / 843)$ & $3.2 \%(\mathrm{n}=33 / 1043)$
\end{tabular}




\begin{tabular}{lll}
\hline & UACP $(\mathbf{n}=\mathbf{8 4 3})$ & BACP $(\mathbf{n}=\mathbf{1 5 7 3})$ \\
\hline COPD & $10.4 \%(\mathrm{n}=78 / 753)$ & $7.7 \%(\mathrm{n}=113 / 1467)$ \\
Chronic Kidney Disease & $10.0 \%(\mathrm{n}=39 / 390)$ & $8.4 \%(\mathrm{n}=38 / 452)$ \\
Previous stroke & $8.4 \%(\mathrm{n}=49 / 582)$ & $10.3 \%(\mathrm{n}=69 / 669)$ \\
Previous CT intervention & $6.1 \%(\mathrm{n}=33 / 539)$ & $7.8 \%(\mathrm{n}=94 / 1207)$ \\
PVD & $15.5 \%(\mathrm{n}=36 / 233)$ & \\
Pre-operative & & \\
characteristics & $15.3 \%(\mathrm{n}=92 / 600)$ & $12.1 \%(\mathrm{n}=28 / 258)$ \\
Hemodynamic instability & $15.9 \%(\mathrm{n}=73 / 458)$ & $16.9 \%(\mathrm{n}=80 / 472)$ \\
Aortic insufficiency & $6.0 \%(\mathrm{n}=36 / 597)$ & $5.5 \%(\mathrm{n}=38 / 697)$ \\
Cerebrovascular symptoms & $53.2 \%(\mathrm{n}=191 / 359)$ & $61.3 \%(\mathrm{n}=212 / 346)$ \\
Malperfusion syndrome & $6.1 \%(\mathrm{n}=28 / 457)$ & $5.8 \%(\mathrm{n}=36 / 625)$ \\
Intubation & & \\
Intra-operative & & $213.0 \pm 67.3(\mathrm{n}=813)$ \\
characteristics & & $138.3 \pm 63.6(\mathrm{n}=1510)$ \\
CPB time (min) & $203.6 \pm 71.5(\mathrm{n}=726)$ & $40.6 \pm 34.4(\mathrm{n}=939)$ \\
Cross Clamp time $(\min )$ & $120.4 \pm 46.8(\mathrm{n}=753)$ & $35.0 \pm 16.2(\mathrm{n}=813)$ \\
UACP and BACP time $(\min )$ & $33.3 \pm 15.6(\mathrm{n}=336)$ & $69.1 \%(\mathrm{n}=1031 / 1492)$ \\
HCA time (min) & $31.0 \pm 12.1(\mathrm{n}=726)$ & $30.9 \%(\mathrm{n}=461 / 1492)$ \\
Hemi-arch repair & $68.9 \%(\mathrm{n}=554 / 789)$ & $24.0 \%(\mathrm{n}=140 / 583)$ \\
Total arch repair & $31.1 \%(\mathrm{n}=245 / 789)$ & $10.9 \%(\mathrm{n}=171 / 1573)$ \\
Bentall procedure & $20.2 \%(\mathrm{n}=100 / 496)$ & $28.9 \%(\mathrm{n}=316 / 1093)$ \\
Concomitant CABG & $8.4 \%(\mathrm{n}=71 / 843)$ & \\
Concomitant AVS & $21.6 \%(\mathrm{n}=97 / 450)$ &
\end{tabular}

All values are presented as mean \pm standard deviation or $\%$ and number; COPD $=$ Chronic Obstructive Pulmonary Disease; CT =cardiothoracic; PVD = Peripheral Vascular Disease; CPB = cardiopulmonary bypass; $\mathrm{HCA}=$ hypothermic circulatory arrest; CABG = Coronary Artery Bypass Graft; AVS = Aortic Valve Surgery

\section{Figure Legends}

Figure 1. Forest plot describing the comparison between UACP and BACP regarding the in-hospital mortality.

Figure 2. Forest plot describing the comparison between UACP and BACP regarding the (A) permanent neurological deficit (PND) and (B) transient neurological deficit (TND).

Supplemental Figure 1. Evaluation of UACP vs. BACP flow diagram.

Supplemental Figure 2. Plot describing the influence of the difference in rates of total arch repair (TAR) between UACP and BACP groups in the rate of permanent neurological deficit (PND) odds ratio.

Supplemental Figure 3. Funnel plots of all assessed outcomes and Egger's test $p$-value. 


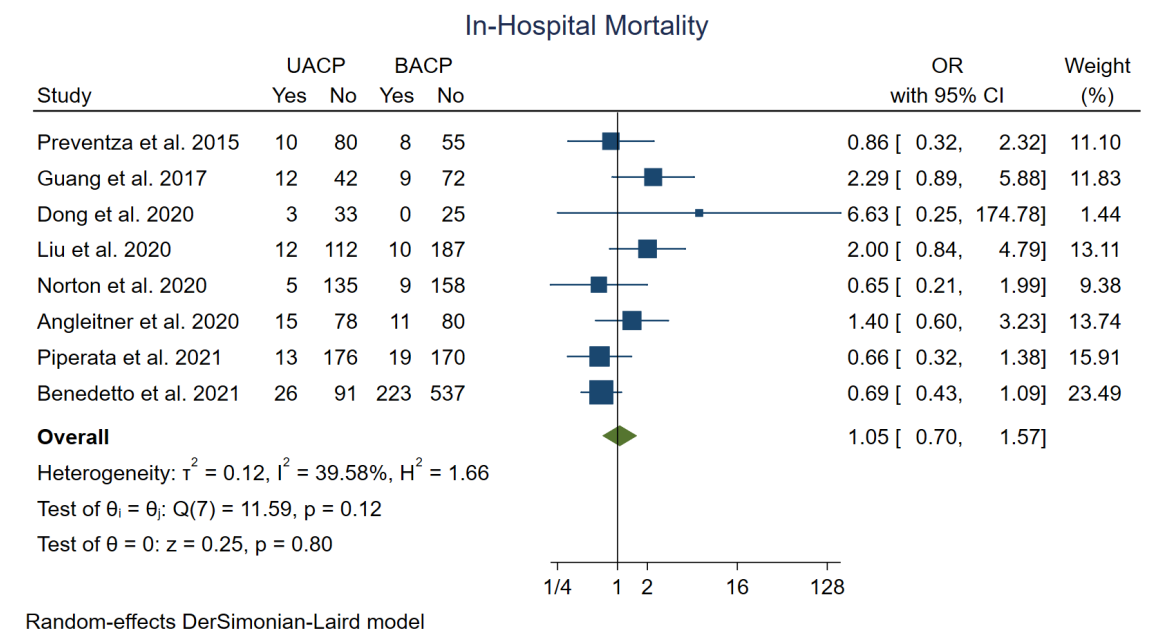

Figure 1. Forest plot describing the comparison between UACP and BACP regarding the in-hospital mortality.

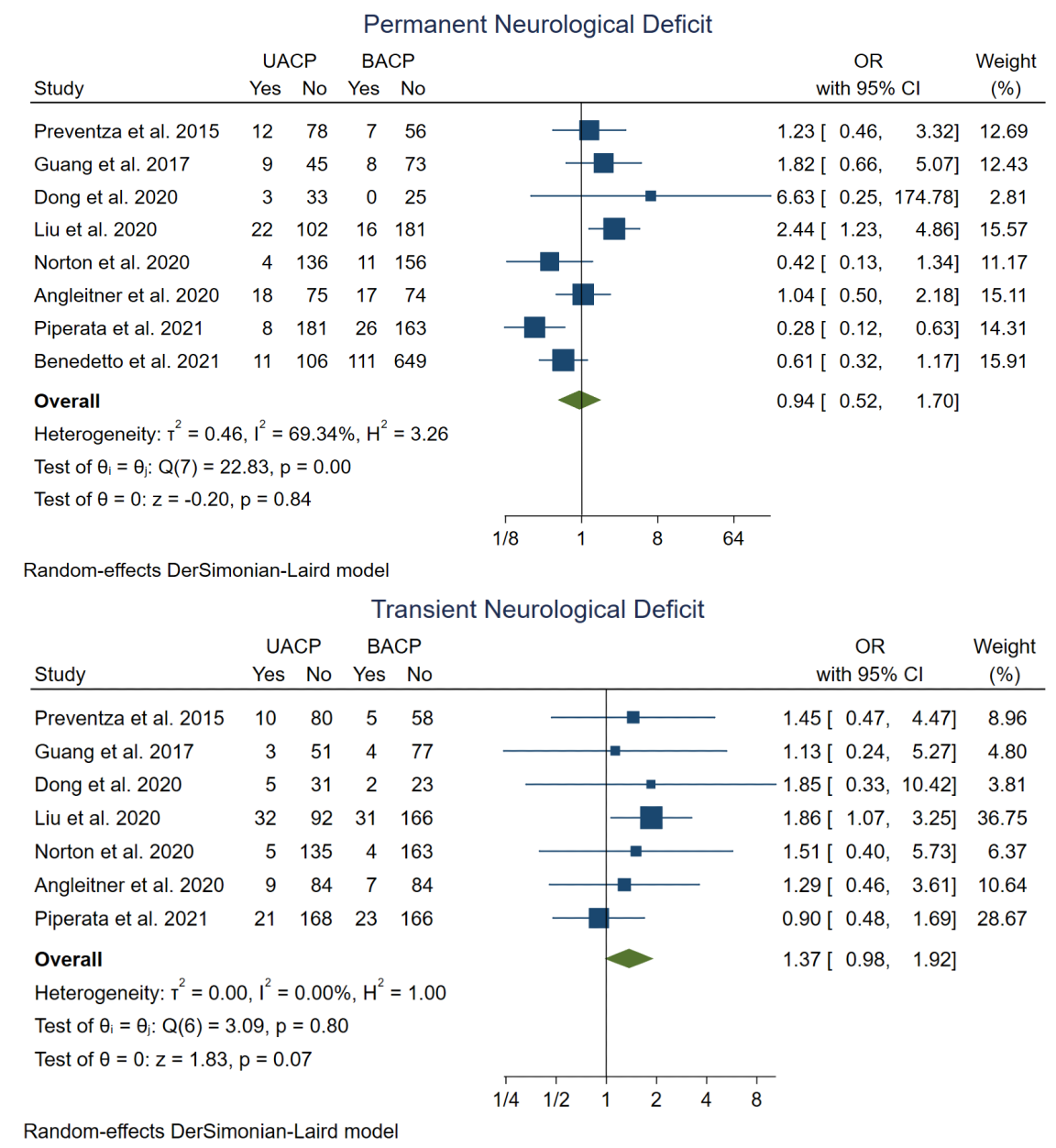

Figure 2. Forest plot describing the comparison between UACP and BACP regarding the (A) permanent neurological deficit (PND) and (B) transient neurological deficit (TND). 


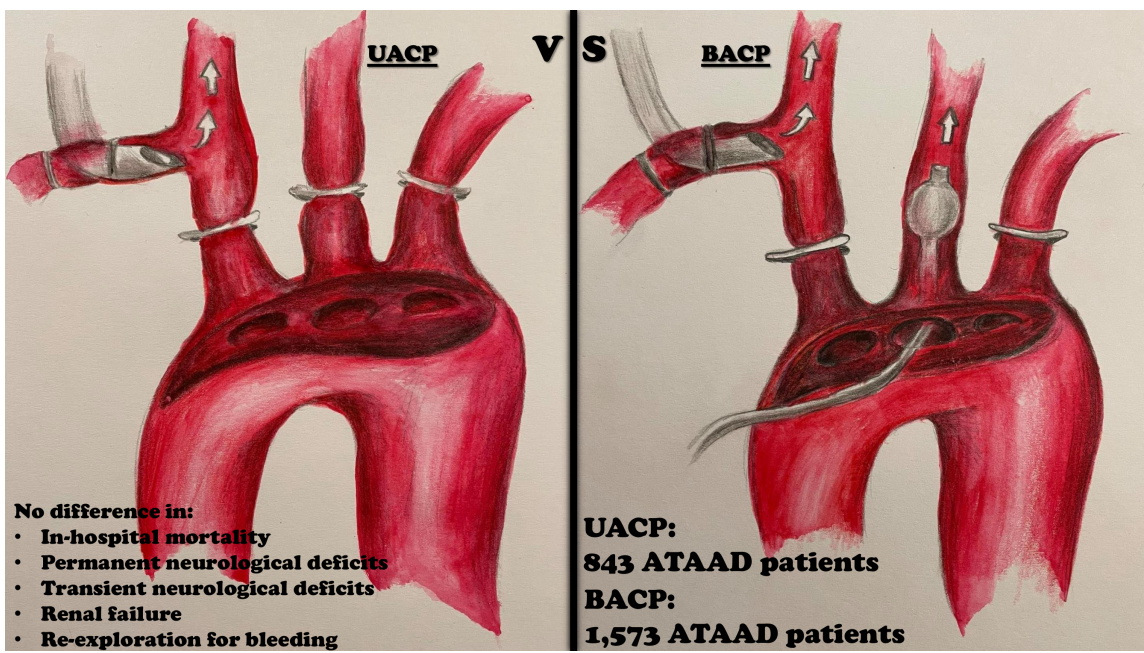

